\title{
Discovering Biomarkers in Gastric Cancer by Urine Metabolomics
}

\section{Yuan Kong}

First Hospital of Jilin University

\section{Hongya Zhang}

First Hospital of Jilin University

\section{Shuang Li}

First Hospital of Jilin University

\section{Jian Suo}

First Hospital of Jilin University

\section{Shaopeng Zhang}

First Hospital of Jilin University

\section{Jian Li}

Maternal and Child Health Hospital of Hubei Province: Hubei Province Women and Children Hospital

\section{Shaokang Yang}

First Hospital of Jilin University

\section{Zhifeng Liu}

First Hospital of Jilin University

\section{Peng Yang}

First Hospital of Jilin University

\section{Qian Li}

First Hospital of Jilin University

Daguang Wang ( $\nabla$ dgwang@jlu.edu.cn )

First Hospital of Jilin University

\section{Research}

Keywords: gastric cancer, metabolomics, biomarkers, diagnosis, urine

Posted Date: August 23rd, 2021

DOI: https://doi.org/10.21203/rs.3.rs-820790/v1

License: (c) (i) This work is licensed under a Creative Commons Attribution 4.0 International License. Read Full License 



\title{
Discovering Biomarkers in Gastric Cancer by Urine Metabolomics
}

Authors : Yuan Kong ${ }^{1 \#}$, Hongya Zhang ${ }^{1 \#}$, Shuang $\mathrm{Li}^{1}$, Jian Suo ${ }^{1}$, Shaopeng Zhang ${ }^{1}{ }_{\text {Jian }}$ $\mathrm{Li}^{2}$, Shaokang Yang ${ }^{1}$, Zhifeng Liu ${ }^{1}$,Peng Yang ${ }^{1}$, Qian Li $^{1}$,Daguang Wang ${ }^{1 *}$.

Affiliation:

1. Department of Gastrointestinal Surgery, the First Hospital of Jilin University, Changchun, Jilin Province, China, 130000.

2. Department of Surgery,Shandong Provincial Maternal and Child Health Care Hospital, Jinan, China, 250000.

Corresponding author: Daguang Wang $\left(^{*}\right)$, Department of Gastrointestinal Surgery, First Hospital of Jilin University, Changchun, Jilin Province, China, 130000. Email address:dgwang@jlu.edu.cn. Tel: 17808068189; Fax: 17808068189;

Yuan Kong, Hongya Zhang contribute equal to this work (\#).

\begin{abstract}
Introduction: Gastric cancer is one of the most common gastrointestinal tumors, ranking forth in incidence and second in mortality worldwide. Discovering molecular biomarkers for early gastric cancer diagnosis is of great importance.

Methods: Urine and related clinical data of 40 patients with gastric cancer (20 in advanced stage and 20 in early stage) and 20 healthy volunteers from Jilin University First Hospital were collected. Liquid chromatography-mass spectrometry (LC-MS) was used to detect urine samples and the metabolic differences between the three groups of urine samples were analyzed. The principal component analysis was performed after data processing, and different metabolites were found using analysis
\end{abstract}


of variance. Partial least square discriminant analysis was performed to further narrow the range of different metabolites. The precise mass to charge ratios of different metabolites were imported into the Human Metabolomics Database (HMDB). Finally, the identified different metabolites were further screened by cluster analysis and ROC curve.

Results: Urine samples of the healthy group (NOR), the early gastric cancer group (EGC), and the advanced gastric cancer group (AGC) were different metabolites. 324 statistically significant metabolites are screened out. The cluster analysis showed 7-Methylguanine, vinylacetylglycine, butyric acid, 4-Vinylphenol sulf, 5 '-biotinyl-AMP, and 3-Amino-2-piperido in EGC, AGC and NGO were similar. 7-Methylguanine, vinylacetylglycine and 4-Vinylphenolsulfate had good diagnostic ability in EGC and NOR $(\mathrm{p}<0.05)$, and gastric cancer and NOR $(\mathrm{p}<0.05)$.

Conclusion: Differences in the metabolites in urine between the early gastric cancer group and the healthy group were found. 7-Methylguanine, Vinylacetylglycine, and 4-Vinylphenolsulfate have good diagnostic ability and may be potential biomarkers of early gastric cancer.

Key words: gastric cancer, metabolomics, biomarkers, diagnosis, urine 


\section{Introduction}

Gastric cancer (GC) is one of the most common gastrointestinal tumors, ranking forth in incidence and second in mortality worldwide[1]. Great progresses have been made in treatment of gastric cancer including surgery, radiotherapy and chemotherapy, and the incidence of gastric cancer is decreasing[2]. However, the 5-year overall survival rate of advanced gastric cancer is still less than $30 \%$, while as high as $95 \%$ for early gastric cancer[3, 4]. Due to the lack of specific symptoms, most patients are diagnosed as advanced gastric cancer characterized by invasion and metastasis when first diagnosed, and have lost the best treatment opportunity[5, 6]. At present, endoscopy-based biopsy and pathological examination are the gold standards for gastric cancer diagnosis but limited to its invasive characteristics. The current clinically commonly used serological tumor markers, such as CEA, CA125 and CA19-9, are with poor sensitivity and specificity for gastric cancer, especially diagnosis and screening of early gastric cancer[7]. Therefore, discovering molecular biomarkers for early gastric cancer diagnosis is of great importance.

Metabolomics has emerged and developed after genomics and proteomics, and has been widely used in the fields of disease diagnosis, biomarker screening, drug efficacy and toxicity evaluation[8]. Currently, metabolomics based on advanced analytical chemistry techniques is regarded as an efficient technical approach for the discovery and screening of relevant molecular markers for early cancer diagnosis and prognosis judgment $[9,10]$. Metabolomics can not only directly and comprehensively 
reflect the changes of endogenous metabolic information in the body, but also obtain biological characteristic metabolic markers after multivariate statistical analysis[11]. In recent years, liquid chromatography, gas chromatography, high-resolution nuclear magnetic resonance spectroscopy, mass spectrometry, and more complex techniques have enabled metabolomics more extensive application in identifying biomarkers that can be used to identify early disease, thereby addressing urgent clinical needs.

Metabolomics has provided solid theoretical support for the discovery of potential biomarkers in different cancers including gastric cancer, lung cancer, et al. Yu et al. aimed to identify the markers of gastric cancer and found several changed materials such as 2-Hydroxybutyrate, pyroglutamate, glutamate etc. in patients with progressive disease or gastric cancer by gas chromatography time-of-flight mass spectrometry of blood plasma[12]. Previous studies have shown that the metabolic activity of cancer cells is significantly different from that of healthy cells. Early detection is the most effective way to improve the prognosis of gastric cancer and the study of metabolic profile helps to identify certain tumor biomarkers and provide a basis for early diagnosis. However, the role of metabolomics in the diagnosis of early gastric cancer is not clear yet.

In this study, liquid chromatography-mass spectrometry (LC-MS) was used to detect urine samples of healthy people, patients with early gastric cancer and patients with advanced gastric cancer. To identify the biomarkers for the diagnosis of early gastric cancer, the metabolic differences between the three groups of urine samples were analyzed. The principal component analysis was performed after data processing, 
and 324 different metabolites were found using analysis of variance. Then, partial least square discriminant analysis was performed to further narrow the range of different metabolites to 11 species. The precise mass to charge ratios of 11 different metabolites were imported into the Human Metabolomics Database (HMDB), and 6 different metabolites were found. Finally, the 6 different metabolites were further screened by cluster analysis and ROC curve.

\section{Methods}

\section{Patients}

A total of 40 patients with gastric cancer (20 in advanced stage and 20 in early stage) and 20 healthy volunteers were included in this study, and their urine and related clinical data were collected. These 40 patients with gastric cancer were admitted to the department of gastrointestinal surgery, Jilin University First Hospital from September 2019 to December 2019. Informed consent was obtained from patients. The study was approved by the Ethics Committee of Jilin University First Hospital and was in compliance with the Declaration of Helsinki.

Patients with a pathological diagnosis of primary GC, between 30 and 70 years old, with good heart, liver and kidney function were included in this study. Patients with congenital diseases, poor general condition, diabetes, other malignant tumors and severe immunosuppressive diseases were excluded.

\section{Sample collection}

The clean mid-section urine was collected, and centrifuged at $3500 \mathrm{r} / \mathrm{min}$ for 5 
minutes. Then, the supernatant is collected and stored at $-80^{\circ} \mathrm{C}$. For sample preparation, the urine sample stored at $-80^{\circ} \mathrm{C}$ was taken out and placed at room temperature for later use. $50 \mu \mathrm{l}$ of each group including early gastric cancer group (EGC), advanced gastric cancer group (AGC), healthy group (NOR) was obtained and mixed together as quality control group. $100 \mu 1$ sample of each group including EGC, AGC, NOR and quality control group (QC) was added into $500 \mu$ lacetonitrile, respectively. After mixing well, the samples were centrifuge at $14000 \mathrm{r} / \mathrm{min}\left(4^{\circ} \mathrm{C}\right)$ for $5 \mathrm{~min}$. The clear supernatant is collected for LC-MS examination.

\section{LC-MS examination}

The LC-MS examination was performed as previously reported[13]. Briefly, the mobile phase was ultrasonicated in a numerically controlled ultrasonic cleaner to exhaust the gas before the LC-MS examination. The, $500 \mu$ sample of each group was chromatographed onto a C18 reverse-phase column $(2.1 \times 100 \mathrm{~mm}, 1.8 \mu \mathrm{m}$, Waters, Milford, MA) using an Agilent 1290 Infinity liquid chromatography system (Agilent, Santa Clara, CA). The system was maintained at $40{ }^{\circ} \mathrm{C}$ for sample separation. The sample elution was carried out at a flow rate of $400 \mu 1 / \mathrm{min}$, with $5 \%$ acetonitrile in water (containing $0.1 \%$ formic acid) for the first $2 \mathrm{~min}$, a linear gradient of $5 \%$ to $95 \%$ acetonitrile over the next $15 \mathrm{~min}$, and $95 \%$ acetonitrile in water (containing $0.1 \%$ formic acid) for the last $2 \mathrm{~min}$.

\section{Statistical Analysis}

The analysis of difference (ANOVA) was used to analyze the data of the positive ion and negative ion mode respectively. The metabolites with statistical differences 
were screened out with $\mathrm{F}$ value $>1$ and $\mathrm{P}<0.05$. The highly specific metabolites with VIP value $>1$ and weight value of $100 \%$ were screened out by partial least squares discrimination analysis (PLS-DA) and support vector machine analysis (SVM). The distribution of metabolites in different groups was determined by cluster analysis. The ROC curves were drawn, and the area under the curve (AUC value) was calculated to evaluate the accuracy of grouping. Then, the mass to charge ratio $(\mathrm{M} / \mathrm{Z})$ of selected metabolites was imported into the Metabolome Database (HMDB) for structural identification to determine the corresponding substances. Finally, the verification group was used to verify the selected differential metabolites, and examine their reliability and sensitivity.

\section{Results}

\section{Different levels of metabolites in urine of gastric cancer patients}

The mass spectrum data of metabolites in urine was obtained by LC-MS and the total ion current (TIC) was drawn (Figure 1A). In three different ion chromatograms, the same retention time corresponded to the same metabolite. It can be seen directly in the ion current diagram that the peaks corresponding to the same retention time of the three groups of samples were significantly different (marked by the arrow), indicating that the urine samples of the NOR, EGC, and AGC were different metabolites. The principal component analysis (PCA) was further performed and significant

differences in the classification of metabolites between groups were observed (Figure 1B). Moreover, 324 statistically significant metabolites are screened out by 
ANOVA using $\mathrm{F}$ value $>1$ and $\mathrm{P}<0.05$ as the criteria, and the differential metabolites in urine samples were selected (Figure 1C).

Figure1
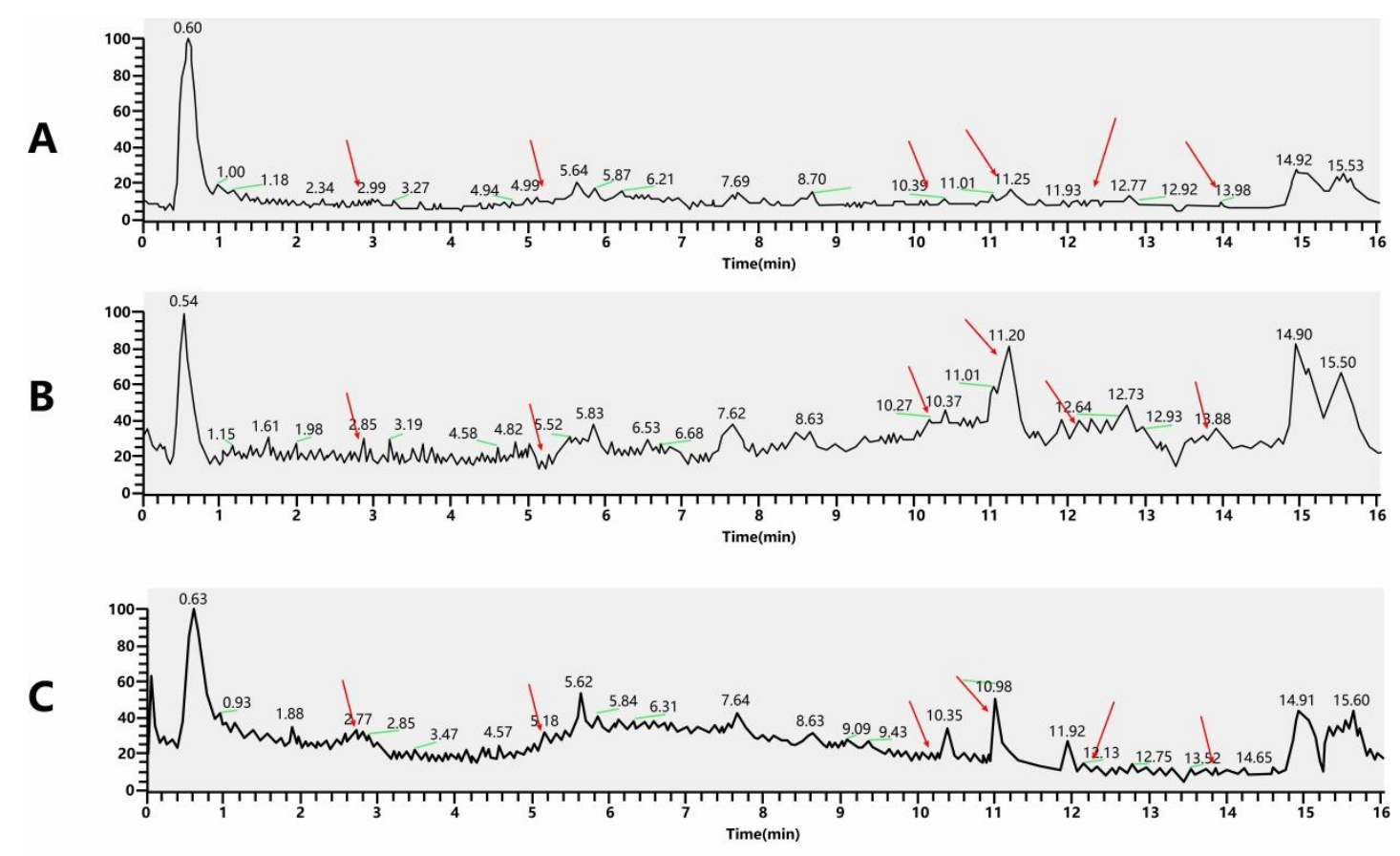

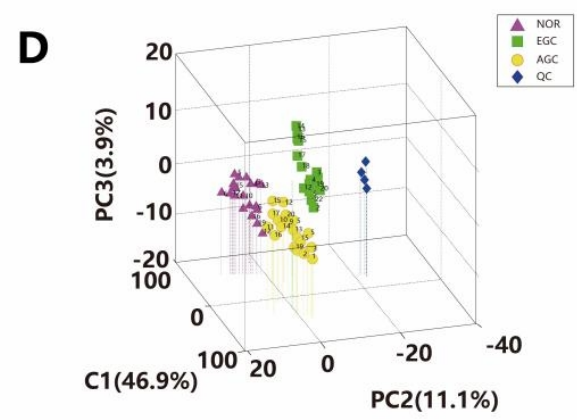

三维主成分分析图

NOR:健康组 EGC:早期胃癌组 AGC:进展期胃癌组 QC:质量控制组

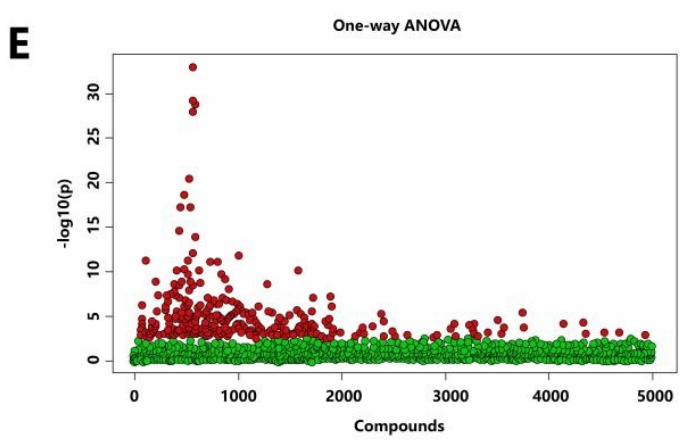

方差分析图

\section{Screening of differential metabolites in urine of gastric cancer patients with a}

\section{weight of $100 \%$}

As shown in Figure 2A, the VIP scores were calculated by PLS-DA and ANOVA.

It was found that a total of 11 metabolites were with VIP value $>1$. Then, a total of 6 different metabolites with a weight of $100 \%$ were found using the SVM in the BRB-array software. After importing the mass to charge ratio $(M / Z)$ into the HMDB 
and comparison, the 6 important differential metabolites are identified (Table 1).

To evaluate the similarity between the three groups of urine samples, the cluster analysis was performed. The result showed 7-Methylguanine, vinylacetylglycine, butyric acid, 4-Vinylphenol sulf, 5 '-biotinyl-AMP, and 3-Amino-2-piperido in EGC, AGC and NGO were similar (Figure 2B).

Table 1 Differential metabolites screened by PLS-DA

\begin{tabular}{llllll}
\hline $\begin{array}{l}\text { Differential } \\
\text { Metabolites }\end{array}$ & $\begin{array}{l}\text { Mass charge } \\
\text { ratio }(\mathrm{m} / \mathrm{z})\end{array}$ & $\begin{array}{l}\text { Retention } \\
\text { time }\end{array}$ & F value & P value & Weight \\
\hline 7-Methylguanine & 198.0985 & 0.61 & 59.438 & $1.13 \mathrm{E}-14$ & 5.6887 \\
3-Amino-piperidon & 114.102 & 0.86 & 7.5885 & 0.001197 & 8.2248 \\
Butyric acid & 121.0859 & 13.88 & 17.251 & $1.39 \mathrm{E}-06$ & 9.8693 \\
5'-biotinyl-AMP & 214.0361 & 8.03 & 11.784 & $5.07 \mathrm{E}-05$ & 2.6442 \\
Vinylacetylglycine & 207.074 & 0.64 & 14.784 & $6.73 \mathrm{E}-06$ & 1.0304 \\
4-Vinylphenolsulfate & 183.0116 & 0.52 & 30.137 & $1.18 \mathrm{E}-09$ & 1.018 \\
\hline
\end{tabular}

Figure2
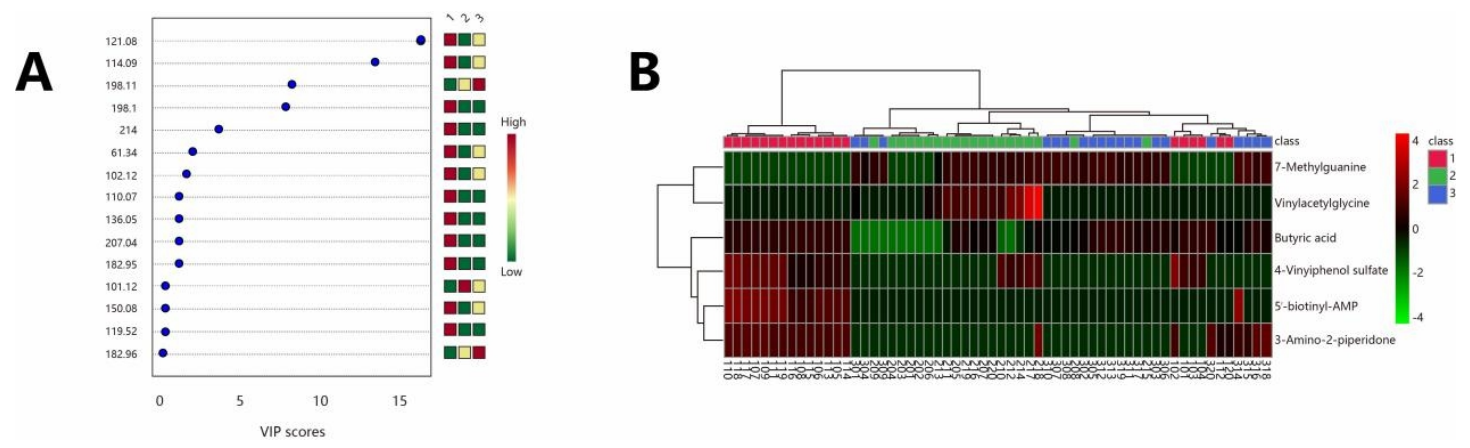

\section{Differential metabolites have good diagnostic ability for patients with early}

\section{gastric cancer}

The ROC curves were drawn and showed that 7-Methylguanine, vinylacetylglycine and 4-Vinylphenolsulfate had good diagnostic ability in EGC and NOR (Figure 3A and Table 2), and gastric cancer and NOR (Figure 3B and Table 3). In comparison, the diagnostic ability of butyric acid, 4-Vinylphenol sulf, 5 '-biotinyl-AMP, and 3-Amino-2-piperido was not satisfied since the AUC was between 0.2 to 0.8 . From the drawn bar chart (Figure 3C), we can see the differences 
in the six different metabolites especially butyric acid in the three groups of samples.

Figure3

A

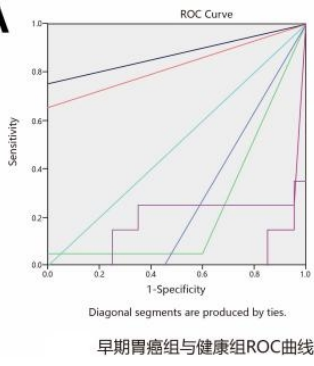

B

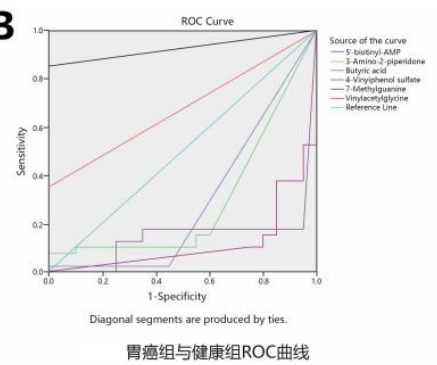

C

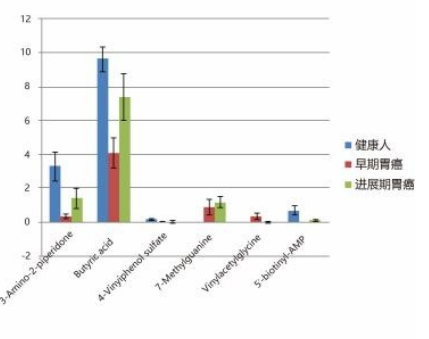

Table 2 ROC analysis in EGC and NOR

\begin{tabular}{llll}
\hline & & \multicolumn{2}{c}{$95 \% \mathrm{Cl}$} \\
\cline { 3 - 4 } Differential & AUC & Lower & Upper \\
Metabolites & & & \\
7-Methylguanine & 0.875 & 0.755 & 0.995 \\
3-Amino-piperidon & 0.240 & 0.082 & 0.398 \\
Butyric acid & 0.330 & 0.053 & 0.079 \\
5'-biotinyl-AMP & 0.250 & 0.048 & 0.447 \\
4-Vinylphenolsulfate & 0.196 & 0.687 & 0.344 \\
Vinylacetylglycine & 0.825 & Table 3 ROC analysis in gastric cancer and NOR \\
\hline & & \multicolumn{2}{c}{0.963} \\
\cline { 3 - 4 } & AUC & Lower & $95 \% \mathrm{Cl}$ \\
Differential & & \multicolumn{2}{c}{ Upper } \\
Metabolites & 0.925 & 0.856 & \\
7-Methylguanine & 0.290 & 0.143 & 0.994 \\
3-Amino-piperidon & 0.114 & 0.027 & 0.437 \\
Butyric acid & 0.293 & 0.140 & 0.201 \\
5'-biotinyl-AMP & 0.147 & 0.044 & 0.447 \\
4-Vinylphenolsulfate & 0.675 & 0.542 & 0.249 \\
Vinylacetylglycine & 0.2808 \\
\hline
\end{tabular}

As shown in Table 4, the sensitivity and specificity of differential metabolites for gastric cancer patients and NOR were $97.5 \%$ and $95 \%$, respectively. As shown in Table 5, the sensitivity and specificity of differential metabolites for EGC and NOR were both $90 \%$. The verification results indicated our experiments have good 
reliability.

Table 4 The sensitivity and specificity of differential metabolites for gastric cancer patients and NOR

\begin{tabular}{|c|c|c|c|c|c|}
\hline \multirow[t]{2}{*}{ Prediction } & \multicolumn{2}{|c|}{ Reality } & \multirow[b]{2}{*}{ Total } & \multirow[b]{2}{*}{ Sensitivity } & \multirow[b]{2}{*}{ Specificity } \\
\hline & $\begin{array}{l}\text { Gastric } \\
\text { cancer }\end{array}$ & NOR & & & \\
\hline $\begin{array}{l}\text { Gastric } \\
\text { cancer }\end{array}$ & 39 & 1 & 40 & & \\
\hline NOR & 1 & 19 & 20 & $97.5 \%$ & $95 \%$ \\
\hline Total & 40 & 20 & 60 & & \\
\hline
\end{tabular}

Table 5 The sensitivity and specificity of differential metabolites for EGC and NOR

\begin{tabular}{|c|c|c|c|c|c|}
\hline \multirow[t]{2}{*}{ Prediction } & \multicolumn{2}{|c|}{ Reality } & \multirow[b]{2}{*}{ Total } & \multirow[b]{2}{*}{ Sensitivity } & \multirow[b]{2}{*}{ Specificity } \\
\hline & EGC & NOR & & & \\
\hline EGC & 18 & 2 & 20 & & \\
\hline NOR & 2 & 18 & 20 & $90 \%$ & $90 \%$ \\
\hline Total & 20 & 20 & 40 & & \\
\hline
\end{tabular}

\section{Discussion}

Gastric cancer, a malignant tumor with high incidence and high mortality, is one of the major health burdens globally, especially in East Asia[14]. Although the incidence rate is decreasing year by year, the incidence of gastric cancer still ranks second among all malignant tumors in China[5]. The mortality rate ranks third in China, among which the mortality rates of men and women rank third and second, respectively[15]. Due to the lack of specific clinical manifestations of EGC, it is usually diagnosed in the late stage[16]. More than $80 \%$ of gastric cancer patients in China are already in the advanced stage at the time of diagnosis. For this reason, many people may miss the opportunity of radical resection and have a high risk of postoperative metastasis and recurrence, resulting in a poor overall prognosis of 
GC[17]. The overall 5-year survival rate of AGC is only about $26 \%$, compared with that of EGC as high as $95 \%[1,4]$. Therefore, early diagnosis, prevention and control of gastric cancer are essential to improve the prognosis of patients and reduce the medical burden[18].

Metabolomics has emerged as a new research field with the advantages of high throughput, high sensitivity and high accuracy, and has broad prospects in the diagnosis, treatment and evaluation of diseases[19]. Metabolomics is a comprehensive study of the metabolome, which is a component of biochemical substances or small molecules that exist in cells, tissues, and body fluids[20]. The application of mass spectrometry coupled with chromatography has greatly improved the efficiency and reliability of metabolic spectrum analysis. In this study, 7-Methylguanine, vinylacetylglycine and 4-Vinylphenolsulfate showed good diagnostic value for gastric cancer and maybe potential biomarkers.

7-Methylguanine is a nucleic acid metabolite that exists in all eukaryotes[21]. 7-Methylguanine in urine is a biomarker of DNA damage caused by methylation[22]. Since DNA methylation can cause gene mutation and cancer, the detection of this biomarker may be of great significance. Studies have found that the content of 7-Methylguanine in malignant tumors such as colon cancer has increased, and its content in the urine of smokers is significantly higher than that of non-smokers[23]. In addition, the content of 7-Methylguanine in urine is positively correlated with smoking, age, and intake of meat, eggs, soybeans, etc., while it is negatively correlated with body weight, BMI, physical activity, and diet index scores[23]. From 
our result, it is found that the level of 7-Methylguanine in urine samples of EGC and AGC was significantly increased, while the content in urine samples of healthy people was very low. the content of 7-Methylguanine in urine showed a gradual increase in healthy people, patients with EGC, and patients with AGC, indicating a distinction among them. Therefore, 7-Methylguanine may be a potential biomarker for the diagnosis of early gastric cancer.

Vinylacetylglycine is a normally minor metabolite of fatty acids. Tanaka et al. has reported the presence of vinylacetylglycine in biological fluids as a clinical diagnosis of disorders of mitochondrial fatty acid beta-oxidation in some patients with congenital metabolic disorders[24]. Bao et al. and Shi et al. used vinylacetylglycine as a marker for fatty acid metabolism[25, 26]. Vinylacetylglycine plays an important role in the normal life activities of the body, but its related research and literature reports on the prognosis of clinical gastric cancer diagnosis and treatment are few. In this experiment, the metabolites in the urine of the EGC group and the healthy group were different. Therefore, Vinylacetylglycine may be a potential biomarker for early gastric cancer and the corresponding specific mechanisms and principles need to be further explored and improved.

4-Vinylphenolsulfate and 2-methoxy-4-vinylphenol (2M4VP) are both 4-vinylphenols, and their physiological effects may be similar. 2M4VP isolated from pine needles showed the anti-inflammatory effect in lipopolysaccharide-stimulated RAW264.7 cells[27]. In addition, 2M4VP effectively reduces the phosphorylation level of focal adhesion kinase (FAK) and AKT, which may be used as a supplement to 
the treatment of pancreatic cancer[28]. In this experiment, the metabolites in urine of 4-Vinylphenolsulfate gastric cancer group and healthy group were different. 4-Vinylphenolsulfate may play an important role in the normal metabolism of the human body and may affect the occurrence and development of gastric cancer.

Butyric acid is a four-carbon fatty acid, one of the short-chain fatty acids produced by the colon, especially the proximal colon microbiota[29, 30]. Butyric acid can not only stimulate the physiological proliferation of normal intestinal epithelial cells, but also inhibit cell proliferation in colon cancer cell lines in vitro by enhancing histone acetylation [31]. Studies have confirmed that Butyric acid can regulate intestinal epithelial transport, improve the inflammation and oxidation state of the intestinal mucosa, strengthen the mucosal barrier, regulate the sensitivity and motility of internal organs, et al[31]. In our experiments, the content of Butyric acid in the urine samples of the healthy group is very high, but is significantly reduced in gastric cancer especially early gastric cancer, which may be related to the decreased appetite and decreased digestion function of gastric cancer patients. This indicates that Butyric acid not only has an inhibitory effect on the onset of colon cancer, but also on the occurrence of gastric cancer. However, Butyric acid may not be a biomarker for gastric cancer because of its bad diagnostic ability.

At present, samples of gastric cancer based on metabolomics are mainly blood, urine, tissue samples, etc. However, the collection of blood and tissue samples is invasive, and the data analysis and biological interpretation of non-invasive urine samples are challenging. The future experiments can also consider analyzing the 
changes of metabolites in saliva and other samples to find its biomarkers. A more comprehensive metabolite analysis should be conducted to study the dynamic change process of the occurrence, development and prognosis of gastric cancer.

\section{Conclusion}

In this study, there are differences in the metabolites in urine between the early gastric cancer group and the healthy group. 7-Methylguanine, Vinylacetylglycine, and 4-Vinylphenolsulfate have good diagnostic ability and may be potential biomarkers of early gastric cancer. This experiment is limited by objective factors such as sample size. To obtain more accurate experimental results, a larger sample size is required. In future research, we will continue to expand the sample size in order to obtain ideal experimental data and give full play to the unique role of metabolomics in tumor occurrence, development, diagnosis and treatment.

\section{Ethics Statement}

The patient sample comes from the First Affiliated Hospital of Jilin University. All patients have the right of written informed consent, and compliance with the declaration of Helsinki.

\section{Disclosure}

The authors declare that they have no conflict of interest. 


\section{References}

1. Siegel, R.L., K.D. Miller, and A. Jemal, Cancer Statistics, 2017. CA Cancer J Clin, 2017. 67(1): p. 7-30.

2. Smyth, E.C., et al., Gastric cancer. Lancet, 2020. 396(10251): p. 635-648.

3. Chen, W., et al., Cancer statistics in China, 2015. CA Cancer J Clin, 2016. 66(2): p. 115-32.

4. Digklia, A. and A.D. Wagner, Advanced gastric cancer: Current treatment landscape and future perspectives. World J Gastroenterol, 2016. 22(8): p. 2403-14.

5. Duarte, H.O., et al., Gastric cancer: Basic aspects. Helicobacter, 2018. 23 Suppl 1: p. e12523.

6. Thrift, A.P. and H.B. El-Serag, Burden of Gastric Cancer. Clin Gastroenterol Hepatol, 2020. 18(3): p. 534-542.

7. Wu, D., et al., Serum biomarker panels for the diagnosis of gastric cancer. 2019. 8(4): p. 1576-1583.

8. Rinschen, M.M. and J. Ivanisevic, Identification of bioactive metabolites using activity metabolomics. 2019. 20(6): p. 353-367.

9. Laíns, I., et al., Metabolomics in the study of retinal health and disease. Prog Retin Eye Res, 2019. 69: p. 57-79.

10. Wishart, D.S., Metabolomics for Investigating Physiological and Pathophysiological Processes. Physiol Rev, 2019. 99(4): p. 1819-1875. 11. Johnson, C.H., J. Ivanisevic, and G. Siuzdak, Metabolomics: beyond biomarkers and towards mechanisms. Nat Rev Mol Cell Biol, 2016. 17(7): p. 451-9. 
12. Yu, L., et al., Metabolomic phenotype of gastric cancer and precancerous stages based on gas chromatography time-of-flight mass spectrometry. J Gastroenterol Hepatol, 2011. 26(8): p. 1290-7.

13. Pan, G., et al., Discovering Biomarkers in Peritoneal Metastasis of Gastric Cancer by Metabolomics. 2020. 13: p. 7199-7211.

14. Molina-Castro, S., et al., Gastric cancer: Basic aspects. Helicobacter, 2017. 22 Suppl 1.

15. Nie, Y., et al., A global burden of gastric cancer: the major impact of China. Expert Rev Gastroenterol Hepatol, 2017. 11(7): p. 651-661.

16. Karimi, P., et al., Gastric cancer: descriptive epidemiology, risk factors, screening, and prevention. Cancer Epidemiol Biomarkers Prev, 2014. 23(5): p. 700-13.

17. Tirino, G., et al., What's New in Gastric Cancer: The Therapeutic Implications of Molecular Classifications and Future Perspectives. 2018. 19(9).

18. Necula, L., et al., Recent advances in gastric cancer early diagnosis. World J Gastroenterol, 2019. 25(17): p. 2029-2044.

19. Jang, C., L. Chen, and J.D. Rabinowitz, Metabolomics and Isotope Tracing. Cell, 2018. 173(4): p. 822-837.

20. Newgard, C.B., Metabolomics and Metabolic Diseases: Where Do We Stand? Cell Metab, 2017. 25(1): p. 43-56.

21. Nilov, D.K., et al., Inhibition of Poly(ADP-Ribose) Polymerase by Nucleic Acid Metabolite 7-Methylguanine. Acta Naturae, 2016. 8(2): p. 108-15.

22. Nilov, D., et al., Molecular Mechanisms of PARP-1 Inhibitor 7-Methylguanine. 
2020. 21(6).

23. Tamae, K., et al., Effect of age, smoking and other lifestyle factors on urinary

7-methylguanine and 8-hydroxydeoxyguanosine. Cancer Sci, 2009. 100(4): p. 715-21.

24. Tanaka, K., et al., Gas-chromatographic method of analysis for urinary organic acids. I. Retention indices of 155 metabolically important compounds. Clin Chem, 1980. 26(13): p. 1839-46.

25. Bao, W., et al., Metabonomic analysis of quercetin against the toxicity of acrylamide in rat urine. 2017. 8(3): p. 1204-1214.

26. Shi, H., et al., Metabolomics analysis of urine from rats administered with long-term, low-dose acrylamide by ultra-performance liquid chromatography-mass spectrometry. Xenobiotica, 2017. 47(5): p. 439-449.

27. Jeong, J.B., et al., Anti-inflammatory effect of 2-methoxy-4-vinylphenol via the suppression of NF-אB and MAPK activation, and acetylation of histone H3. Arch Pharm Res, 2011. 34(12): p. 2109-16.

28. Kim, D.H., et al., 2-Methoxy-4-vinylphenol Attenuates Migration of Human Pancreatic Cancer Cells via Blockade of FAK and AKT Signaling. Anticancer Res, 2019. 39(12): p. 6685-6691.

29. He, B. and R. Moreau, Lipid-regulating properties of butyric acid and 4-phenylbutyric acid: Molecular mechanisms and therapeutic applications. Pharmacol Res, 2019. 144: p. 116-131.

30. Cueno, M.E. and K. Ochiai, Re-discovering periodontal butyric acid: New insights on an old metabolite. Microb Pathog, 2016. 94: p. 48-53. 
31. Sossai, P., Butyric acid: what is the future for this old substance? Swiss Med Wkly, 2012. 142: p. w13596. 


\section{Figure legend}

Figure 1. (A) Total ion current of healthy group (NOR; a), gastric cancer group (EGC; b), and advanced gastric cancer group (AGC; c). The ordinate represents the relative kurtosis of the metabolites, and the abscissa represents the retention time of the metabolites. (B) The principal component analysis of NOR, EGC, AGC and QC (Quality control). (C) One-way ANOVA analysis. The difference metabolites screened out are shown in red, which can be considered as meaningful metabolites.

Figure 2. (A) Partial least squares discrimination analysis. 1: NOR, 2: EGC, 3: AGC. The abscissa represents the weight value, and the ordinate represents the mass to charge ratio. (B) Dendrogram of cluster analysis of different metabolites. 1: NOR, 2: EGC, 3: AGC. The number below is the number of each urine sample, and the tree-like structure above represents the settlement relationship of similarity between the urine samples. The color of each rectangle is determined by the content of metabolites in the urine sample varying from red (high content) to green (low content).

Figure 3. (A) ROC curve analysis of six metabolites in EGC and NOR. (B) ROC curve analysis of six metabolites in EGC and NOR. (C) Content of six metabolites in NOR, EGC and AGC. 


\section{Tables}

Table 1 Differential metabolites screened by PLS-DA

\begin{tabular}{llllll}
\hline $\begin{array}{l}\text { Differential } \\
\text { Metabolites }\end{array}$ & $\begin{array}{l}\text { Mass } \\
\text { charge } \\
\text { ratio (m/z) }\end{array}$ & $\begin{array}{l}\text { Retention } \\
\text { time }\end{array}$ & F value & P value & Weight \\
\hline 7-Methylguanine & 198.0985 & 0.61 & 59.438 & $1.13 \mathrm{E}-14$ & 5.6887 \\
3-Amino-piperidon & 114.102 & 0.86 & 7.5885 & 0.001197 & 8.2248 \\
Butyric acid & 121.0859 & 13.88 & 17.251 & $1.39 \mathrm{E}-06$ & 9.8693 \\
5'-biotinyl-AMP & 214.0361 & 8.03 & 11.784 & $5.07 \mathrm{E}-05$ & 2.6442 \\
Vinylacetylglycine & 207.074 & 0.64 & 14.784 & $6.73 \mathrm{E}-06$ & 1.0304 \\
4-Vinylphenolsulfate & 183.0116 & 0.52 & 30.137 & $1.18 \mathrm{E}-09$ & 1.018 \\
\hline
\end{tabular}


Table 2 ROC analysis in EGC and NOR

\begin{tabular}{llll}
\hline & & \multicolumn{2}{l}{$95 \%$ CI } \\
\cline { 3 - 4 } $\begin{array}{l}\text { Differential } \\
\text { Metabolites }\end{array}$ & AUC & Lower & Upper \\
7-Methylguanine & 0.875 & 0.755 & 0.995 \\
3-Amino-piperidon & 0.240 & 0.082 & 0.398 \\
Butyric acid & 0.330 & 0.00 & 0.079 \\
5'-biotinyl-AMP & 0.250 & 0.053 & 0.447 \\
4-Vinylphenolsulfate & 0.196 & 0.048 & 0.344 \\
Vinylacetylglycine & 0.825 & 0.687 & 0.963 \\
\hline
\end{tabular}


Table 3 ROC analysis in gastric cancer and NOR

\begin{tabular}{llll}
\hline & & \multicolumn{2}{c}{$95 \%$ CI } \\
\cline { 3 - 4 } $\begin{array}{l}\text { Differential } \\
\text { Metabolites }\end{array}$ & AUC & Lower & Upper \\
7-Methylguanine & 0.925 & 0.856 & 0.994 \\
3-Amino-piperidon & 0.290 & 0.143 & 0.437 \\
Butyric acid & 0.114 & 0.027 & 0.201 \\
5'-biotinyl-AMP & 0.293 & 0.140 & 0.447 \\
4-Vinylphenolsulfate & 0.147 & 0.044 & 0.249 \\
Vinylacetylglycine & 0.675 & 0.542 & 0.808 \\
\hline
\end{tabular}


Table 4 The sensitivity and specificity of differential metabolites for gastric cancer patients and NOR

\begin{tabular}{llllll}
\hline Prediction & Reality & & & \\
& $\begin{array}{l}\text { Gastric } \\
\text { cancer }\end{array}$ & NOR & Total & Sensitivity & Specificity \\
\hline Gastric & 39 & 1 & 40 & & \\
cancer & & 19 & 20 & $97.5 \%$ & $95 \%$ \\
NOR & 1 & 20 & 60 & & \\
Total & 40 & & & & \\
\hline
\end{tabular}


Table 5 The sensitivity and specificity of differential metabolites for EGC and NOR

\begin{tabular}{llllll}
\hline Prediction & Reality & & & \\
\cline { 2 - 3 } & EGC & NOR & Total & Sensitivity & Specificity \\
\hline EGC & 18 & 2 & 20 & & \\
NOR & 2 & 18 & 20 & $90 \%$ & $90 \%$ \\
Total & 20 & 20 & 40 & & \\
\hline
\end{tabular}

